\title{
Are all Non-sustained Ventricular Tachycardia the Same in Hypertrophic Cardiomyopathy Risk Stratification for Sudden Cardiac Death?
}

\author{
Khaled Sabeh ${ }^{1}$ and Marwan Refaat ${ }^{2}$ \\ ${ }^{1}$ Massachusetts General Hospital \\ ${ }^{2}$ American University of Beirut Medical Center
}

May 18, 2020

\author{
Are all Non-sustained Ventricular Tachycardia the Same in Hypertrophic Cardiomyopathy \\ Risk Stratification for Sudden Cardiac Death? \\ Mohamad Khaled Sabeh $\mathrm{MD}^{1}$, Marwan M. Refaat MD ${ }^{2}$ \\ ${ }^{1}$ Cardiac Arrhythmia Service, Massachusetts General Hospital, Boston, Massachusetts - USA \\ ${ }^{2}$ Division of Cardiology, Department of Internal Medicine, American University of Beirut Medical Center \\ Beirut, Lebanon \\ Running Title: NSVT in HCM SCD Risk Stratification \\ Words (excluding references): 664 \\ Disclosures: None \\ Funding: None
}

Keywords: Hypertrophic Cardiomyopathy, Non-sustained Ventricular Tachycardia, Cardiac Arrhythmias, Cardiovascular Diseases

Correspondence:

Marwan M. Refaat, MD, FACC, FAHA, FHRS, FASE, FESC, FACP, FRCP

Associate Professor of Medicine

Director, Cardiovascular Fellowship Program

Department of Internal Medicine, Cardiovascular Medicine/Cardiac Electrophysiology

Department of Biochemistry and Molecular Genetics

American University of Beirut Faculty of Medicine and Medical Center

PO Box 11-0236, Riad El-Solh 1107 2020- Beirut, Lebanon

Fax: +961-1-370814

Clinic: +961-1-350000/+961-1-374374 Extension 5800

Office: +961-1-350000/+961-1-374374 Extension 5353 or Extension 5366 (Direct)

Email: mr48@aub.edu.lb 
Cardiomyopathies with reduced systolic function predispose to sudden cardiac death (SCD) and many studies helped in decreasing that risk by Implantable Cardioverter Defibrillator (ICD) implantation and pharmacologic management (1-4). Many types of cardiomyopathies with preserved systolic function, including hypertrophic cardiomyopathy $(\mathrm{HCM})$, can predispose to malignant ventricular arrhythmias and SCD. HCM is the most common inherited cardiac disease that affects 1 in 200 live births $(5,6)$. SCD remains one of the main causes of death in HCM and the SCD rate peaks in early adulthood (7-14). Data from ICDs suggest that SCD in HCM is most commonly caused by ventricular fibrillation (VF) (15). One major clinical challenge is identifying patients at risk for SCD. Multiple studies showed that non-sustained ventricular tachycardia (NSVT) is a risk actor for SCD $(16,17)$. However the strength of the data was variable across these studies due to difference in populations and the low sensitivity of Holter ECG. Moreover, other studies looked at the rate and duration of the ventricular arrhythmias and their relationship to SCD in HCM (17-19) yet the effect of the morphology of NSVT on SCD has not been well investigated.

In this single center study Adduci et al . explore the prognostic impact of different NSVT morphologies in a cohort of 109 consecutive HCM patients. The study included patients who had an ICD implanted in the authors' institution from January 2001 to December 2018. The ICDs were mostly implanted for primary prevention in HCM patient with 1) one or more risk factor including maximal LV thickness [?]30 mm, family history of SD in at least 1 first-degree relative $<50$ years of age, non-sustained ventricular tachycardia (NSVT), recent ([?] 6 months) unexplained syncope, 2) hypotensive blood pressure during exercise with at least one additional major risk factor for SD 3) end-stage HCM regardless of other established risk markers of SCD. Devices were interrogated on evaluation every 3 to 6 months and the data was assessed for appropriate or inappropriate ICD therapies. Two independent electrophysiologists analyzed the ICD near field and far field EGMs from the ventricular tachycardia runs. They classified the VTs as either monomorphic (MMVT) or polymorphic (PMVT).

During a mean follow up of 71+/- 48 months, 377 NSVT episodes of NSVT were retrieved from ICD memory in 46 patients; of these episodes, $7(2 \%)$ were polymorphic and $370(98 \%)$ were monomorphic (MM). The mean HR of The MM NSVT had an average HR of $171+/-32$ BPM and lasted for $17+/-12$ beats while the PMVT were faster at 241BPM +/- and longer at $28+/-16$ beats. The appropriate intervention rate was $5.1 \%$ per year and interestingly NSVT did not predict the occurrence of ICD therapy. However patients with polymorphic NSVT had a statistically higher risk for ICD intervention as compared to monomorphic NSVT. Further analysis noted a trend for increased risk of ICD therapy with patients with >1 NSVT morphology. Moreover $75 \%$ of the treated VTs had been previously observed as NSVT.

Risk stratification is very important in this young patient population; decreasing the risk threshold for ICD implants leads to missed arrhythmias and bad outcomes while increasing it increases the risk for complications from unnecessarily implanted devices. There are several types of ICDs: Transvenous ICD, Subcutaneous ICD and Extravascular ICD. The results of this study suggest that the risk of SCD in patients with PMVT and/or NSVT with multiple morphologies is different from that of patients with a MMVT, and that the presence of short MMVT doe not predict the future ICD therapies. As such, one may consider a conservative approach in low-risk patients with short bursts of slow MM NSVT, and a more aggressive approach in patients with frequent, rapid rate burst of PMVT. Although this study suggests that different NSVT morphologies affect the prognosis in HCM patients, the low number of events lacked the statistical power to redefine ICD candidacy. Larger multicenter studies are needed to confirm these findings and to help delineate the "at risk patients" who would truly benefit from ICDs.

\section{References:}

1. El Moheb M, Nicolas J, Khamis A, Akl EA, Refaat MM. Cochrane corner: implantable cardiac defibrillators for patients with non-ischaemic cardiomyopathy. Heart. 2020 May;106(9):636-638.

2. Wachter R, Senni M, Belohlavek J, Straburzynska-Migaj E, Witte KK, Kobalava Z, et al. Initiation of sacubitril/valsartan in haemodynamically stabilised heart failure patients in hospital or early after discharge: primary results of the randomised TRANSITION study. Eur J Heart Fail. 2019; 21 (8): 998-1007. 
3. El Moheb M, Nicolas J, Khamis AM, Iskandarani G, Akl EA, Refaat M. Implantable cardiac defibrillators for people with non-ischaemic cardiomyopathy. Cochrane Database Syst Rev. 2018 Dec 8;12:CD012738.

4. AlJaroudi WA, Refaat MM, Habib RH, Al-Shaar L, Singh M, Gutmann R, et al.. Effect of Angiotensin Converting Enzyme Inhibitors and Receptor Blockers on Appropriate Implantable Cardiac Defibrillator Shock: Insights from the GRADE Multicenter Registry. Am J Cardiol Apr 2015; 115 (7): 115(7):92431.

5. Maron BJ, Ommen SR, Semsarian C, Spirito P, Olivotto I, Maron MS. Hypertrophic cardiomyopathy: present and future, with translation into contemporary cardiovascular medicine. J Am Coll Cardiol 2014;64:83-99

6. Semsarian C, Ingles J, Maron MS, Maron BJ. New perspectives on the prevalence of hypertrophic cardiomyopathy. J Am Coll Cardiol 2015;65:1249-54

7. Maron BJ, Olivotto I, Spirito P, Casey SA, Bellone P, Gohman TE, et al. Epidemiology of hypertrophic cardiomyopathy-related death: revisited in a large non-referral-based patient population. Circulation 2000;102:858-64.

8. Maron BJ, Rowin EJ, Casey SA, Link MS, Lesser JR, Chan RH, et al. Hypertrophic cardiomyopathy in adulthood associated with low cardio- vascular mortality with contemporary management strategies. J Am Coll Cardiol 2015;65:1915-28.

9. Bagnall RD, Weintraub RG, Ingles J, Duflou J, Yeates L, Lam L, et al. A prospective study of sudden cardiac death among children and young adults. N Engl J Med 2016;374:2441-52.

10. Elliot PM, Gimeno JR, Thaman R, et al. Historical trends in reported survival rates in patients with hypertrophic cardiomyopathy. Heart 2006;92:785-91.

11. O'Mahony C, Esteban MTT, Lambiase PD, et al. A validation study of the 2003 American College of Cardiology/European Society of Cardiology and 2011 American College of Cardiology Foundation/ American Heart Association risk stratification and treatment algorithms for sudden cardiac death in patients with hypertrophic cardiomyopathy. Heart 2013;99:534-41.

12. Refaat MM, Fahed AC, Hassanieh S, Hotait M, Arabi M, Skouri H, Seidman JG, Seidman CE, Bitar FF, Nemer G: The Muscle Bound Heart. Card Electrophysiol Clin Mar 2016; 8(1): 223-31.

13. Refaat MM, Hotait M, London B: Genetics of Sudden Cardiac Death. Curr Cardiol Rep Jul 2015; 17(7): 606 .

14. Refaat MM, Hotait M, Tseng ZH: Utility of the Exercise Electrocardiogram Testing in Sudden Cardiac Death Risk Stratification. Ann Noninvasive Electrocardiol 2014; 19(4): 311-318.

15. Elliott PM, Gimeno JR, Tome MT, et al. Left ventricular outflow tract obstruction and sudden death risk in patients with hypertrophic cardiomyopathy. Eur Heart J 2006;27:1933-41.

16. Maron BJ, Savage DD, Wolfson JK, Epstein SE. Prognostic significance of 24 hour ambulatory electrocardiographic monitoring in patients with hypertrophic cardiomyopathy: A prospective study. Am J Cardiol. 1981;48(2):252-7.

17. Monserrat L, Elliott PM, Gimeno JR, Sharma S, Penas-Lado M, McKenna WJ. Non- sustained ventricular tachycardia in hypertrophic cardiomyopathy: An independent marker of sudden death risk in young patients. J Am Coll Cardiol. 2003;42(5):873-9.

18. Adabag AS, Casey SA, Kuskowski MA, Zenovich AG, Maron BJ. Spectrum and prognostic significance of arrhythmias on ambulatory Holter electrocardiogram in hypertrophic cardiomyopathy. J Am Coll Cardiol. 2005;45(697-704).

19. Francia P, Santini D, Musumeci B, Semprini L, Adduci C, Pagannone E, Proietti G, et al. Clinical impact of nonsustained ventricular tachycardia recorded by the implantable cardioverter-defibrillator in patients with hypertrophic cardiomyopathy. J Cardiovasc Electrophysiol. 2014;25(11):1180-7. 